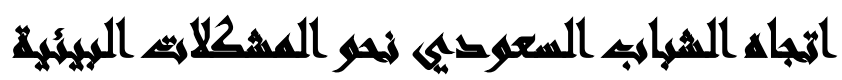

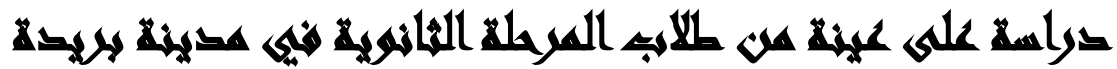

[ᄉ]

حاتم عبد المنعم(')- مصطفى إبراهيم عوض(") - أحمد محمد التويجري(")

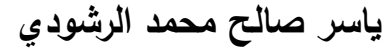

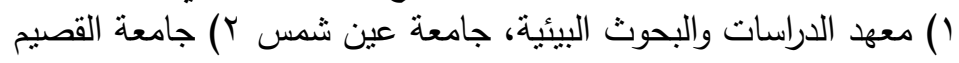

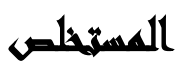

تعد الاتجاهات وفق رأي العلماء من اهم مكونات الخبرة التي تحدد مدي قدرة الفرد علي لطاي

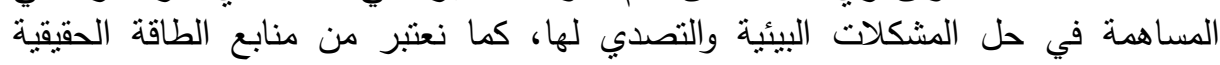

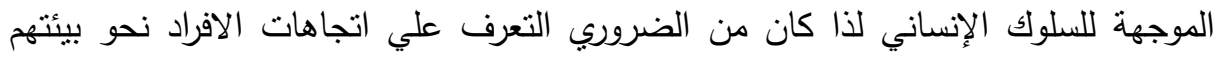

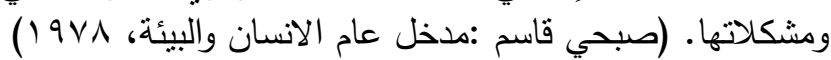

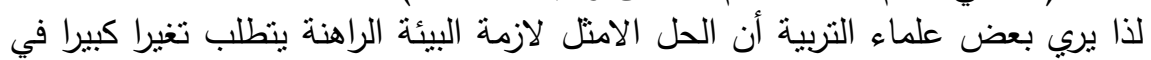

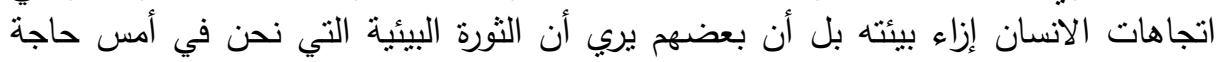

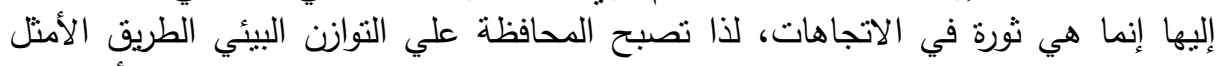

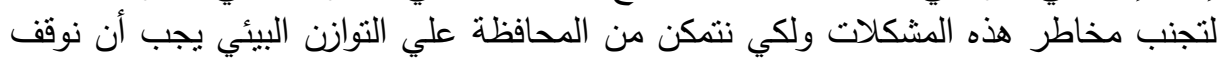
التدخل الجائر للإنسان في الانثطة البيئية.

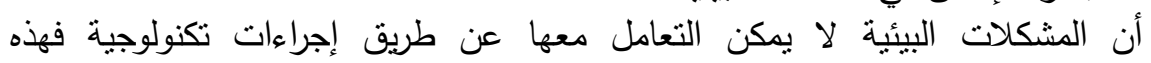

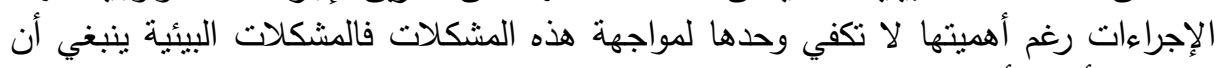

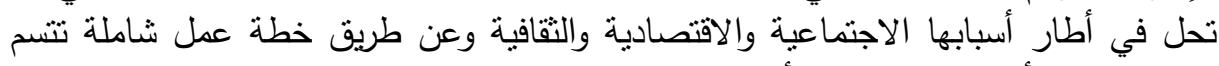
الانضباط في أنماط التنمية وفي أساليب حياة الإفراد وفي سلوكهم وهناك العديد من المشكلات

وقد أدرك الدهتمون بقضايا البيئة ومشكلاتها كما أدرك علماء التربية أن من أهم الطرق

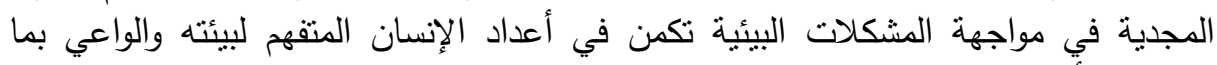

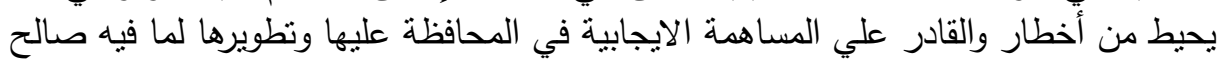

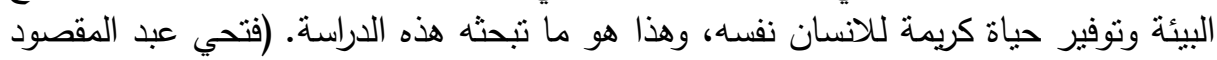

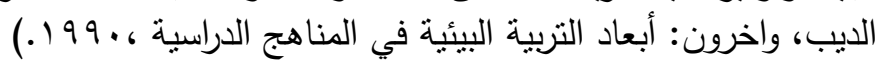

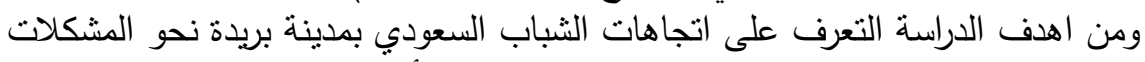

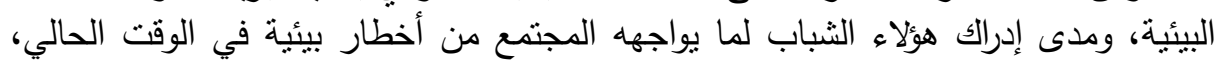
ورؤية هؤلاء الثباب لكيفية التظلب على هذه المشاكل

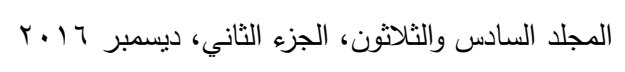
177 


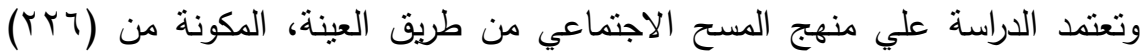
طالب وطالبة من مدارس منطقة بريدة من السعودية وان أداء أداة البحث المستخدمة هي استمارة استبيان للمشكلات البيئية

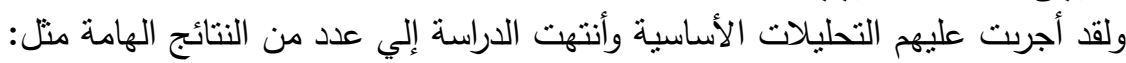

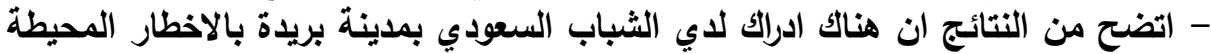
به الناتجة عن المشكلات البيئية ومنها:

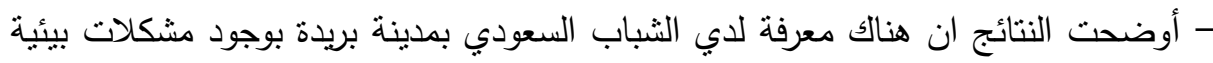

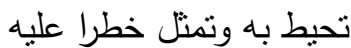
- أوضحت النتائج ان هناك اتجاهات من كلا الجنسين من الثباب تجاه الششكلات التي

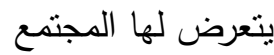
وكذلك عدد من التوصيات منها: - أن تشنتل المناهج الدراسية علي مشكلات بيئية واقعية يمكن عرضها بطريقة مناسبة

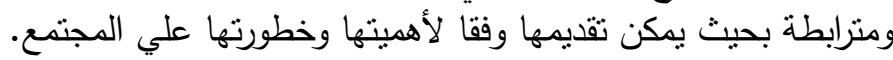
- زيادة فرص النشاط البيئي في مناهج المواد الدراسية المختلفة لممارستها داخل المنان المدرسة

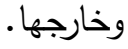

\section{Xas}

التغير صفة اساسية من صفات المجتمع البشري وهي دلالة من دلالات الوجود الانساني

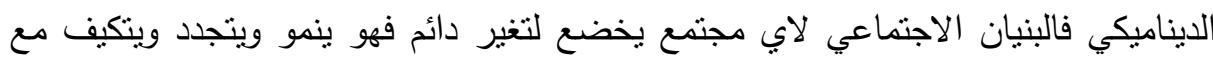

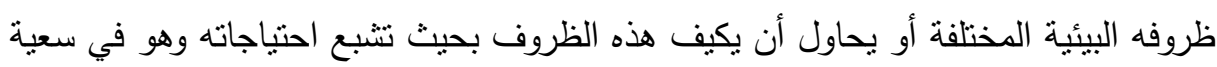
هذا قد يعرض البيئة المحيطة به للكثير من المشكلات التي من كثرة ما ألفها وتعود عليها أصبح يجهل أنها مشكلات بيئية وهو بذلك يدمر نفسه قبل أن يدمر البيئة المحيطة به.

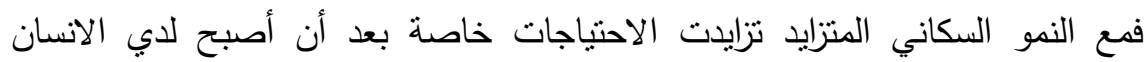
وسائل تكنولوجية منطورة زادت من مقدرته علي التحكم في ظروف البيئة وفي استنغلال مواردها، وقد أمعن الإنسان في استغلال الموارد دون مراعاة نوازن البيئة واحتياجات الكائنات

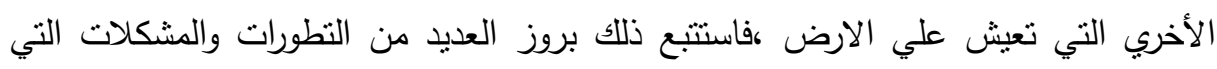

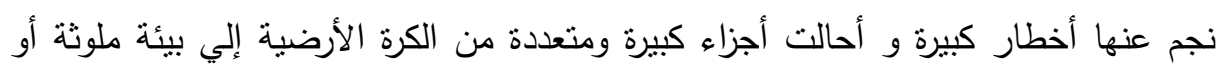
ذات نوعية رديئة تكاد لا تصلح لحياة شتي أنواع الإحياء وفي كثير من المناطق تردت أحوال 
البيئة إلي درجة أصبحت فيها حياة الإنسان نفسه مهدده بالخطر · (صبحي قاسم: مدخل عام

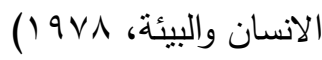

لقد أصبحت البيئة في أنحاء عديدة من العالم غير قادرة علي حفظ نوازنها الطبيعي نتيجة لتزايد المشكلات البيئية ومنها مشكلات التلوث والتتويه واسنتزاف الموارد الطبيعية المتجددة وغير المتجددة وتأكل الثواطئ والتصحر والقصور في موارد الطاقة وسوء التخطيط البيئي والانفجار السكاني • (صبري الدمرداش، محمد احد الدسوقي مقياس الاتجاهات البيئية

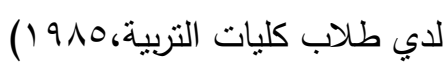
لقد تدخل الإنسان في البيئة بلا روية ولا إدراك لرفاهيم النظام العام الذي يحكم هذا

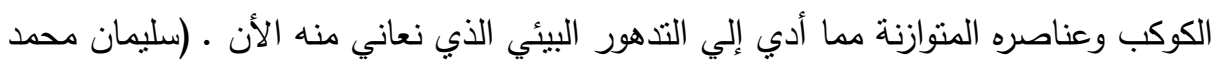

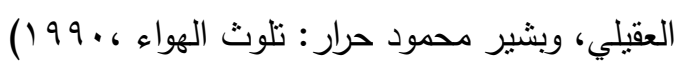
لذلك تصبح المحافظة علي التوازن البيئي الطريق الأمثل لتجنب مخاطر هذه المشكلات ولكي نحافظ علي التوازن البيئي يجب أن نوقف التدخل الجائر للإنسان في الانثطة البيئية.

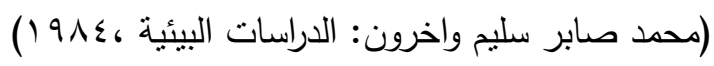

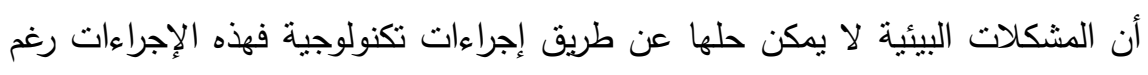

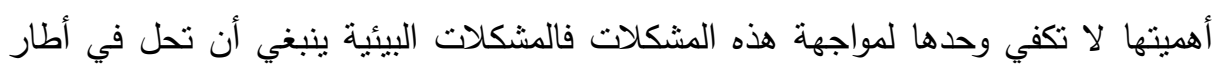

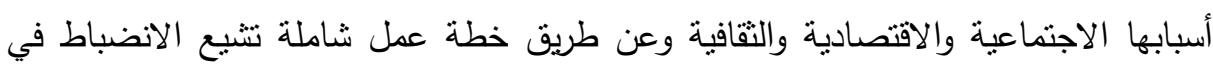
أنماط التنمية وفي أساليب حياة الافراد وفي سلوكهم. وهناك العديد من المشكلات البيئية ومنها تلوث الماء نتيجة وجود مواد كيميائية خطرة او لون ما تتعرض له المياه من تلوث حراري ناتج عن النفايات الصناعية او تلوث التربة بالنفايات

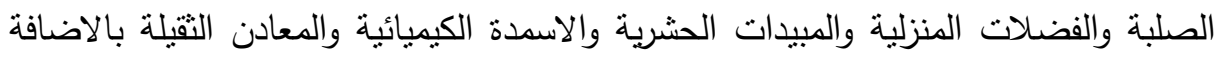
الي التلوث الصوتي والضوضائي. أما النلوث النفطي فيعد من أكثر الملوثات شيوعا في المجتمع وأثندها خطرا علي البيئة بوجه عام والحياة المائية بوجه خاص،ئما التلوث الغذائي الطبيعي الناتج عن تحلل الغذاء بسبب البكتريا او الفطريات او طول فترة التخزين او التعرض للاشعاع الطبيعي وغيره من بن

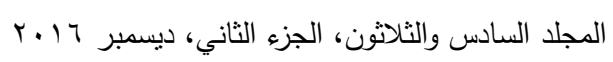


العوامل التي لا يكون الانسان سببا فيها وهناك تلوث غير طبيعي للغذاء ناجم أساسا عن

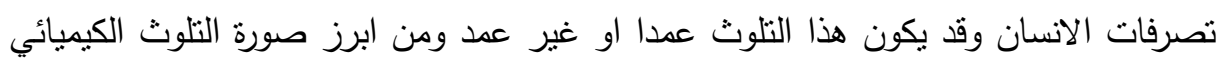
للاغذية.(وائل منصور أحمد برهوم: المشكلات البيئية المتضمنة في محتوى كتاب الجغرافيا-

ومن هنا برزت أهمية هذه الدراسة في التعرف علي مدي إدرالك الثباب السعودي بمدينة

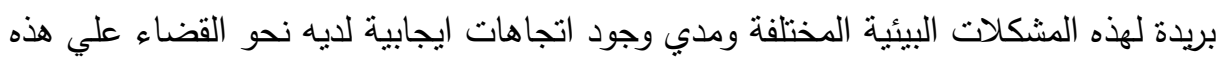
المشكلات او اتجاهات سلبية تزيد من خطورة هذه المشكلات وترسيخ وجودها، وما هي لهي الوسائل الني يري الثباب السعودي أنها يمكن ان تسهم في التغلب علي هذه المشكلات البيئية المنتوعة

أن حل هذه المشكلات يجب أن تتبع أساسا من فهم و إدراك طبيعية العلاقة بين الأنسان والبيئة والجوانب غير الصحيحة في هذه العلاقة حتي يمكن معالجتها علي أسس سليمة، فلكي

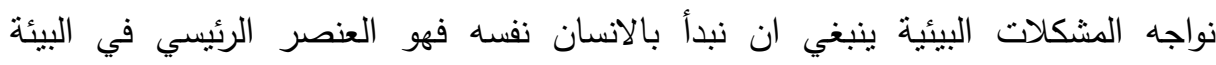
والمستقبد منها والسبب المباثر في مشاكلها وعلي هذا الاساس فانه مهما صدرت التشريعات وتأسست الهيئات والجمعيات و وعندت المؤتمرات الدولية الخاصة بحماية البيئة فلن تؤدي الي ضمان السلوك التئس السليم من قبل الإفراد

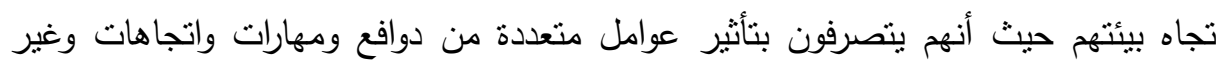
ذلك، إن الاساس في ذلك هو العنصر التربوي بالدرجة الأولي. (رياض الجبان، عبد الحميد دوني

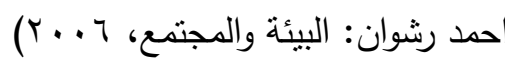
وقد أدرك المهتمون بقضايا البيئة ومشكلاتها كما أدرك المربون أن من أهم الطرق

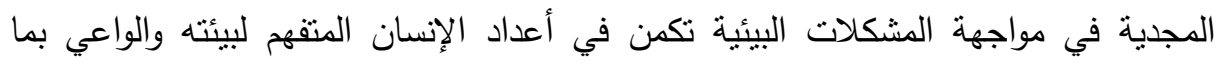
يحيط به من أخطار والقادر علي المساهمة الايجابية في المحافظة عليها وتطويرها لما فيه صالح البيئة وتوفير حياة كريمة للانسان نفسه.ومن المرتمون من الدراسات السابقة 
دراسة "أسماء محمود غانم وآخرون(r . . †): بعنوان "فاعلية برامج حماية البيئة بالمجلس الأعلى للثباب والرياضة لتتمية الاتجاهات البيئية لدى الطلائع "

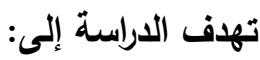
- التعرف على مدى فاعلية برامج حماية البيئة بالمجلس الأعلى للشباب والرياضة على تتمية الاتجاهات البيئية لاى الطلائع. - - إلقاء الضوء على إسهامات جماعات البيئة في المجتمع في التعرف على أوجه القصور في البرامج المقدمة ومعالجتها. دراسة فادية مغيث( •99 ( 9 ): تهدف هذه الدراسة إلى تحديد دور التربية بمؤستها

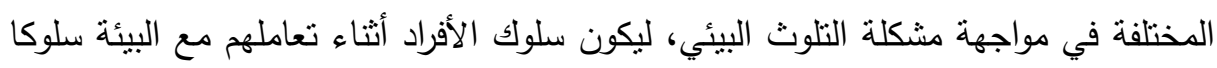
إيجابيا. وقد استخدم في هذه الدراسة الأدوات الآتية: الاستبيان وقد تكونت عينة الدراسة من Vجq تلميذا من مراحل التعليم الابتدائي والإعدادي والثانوي

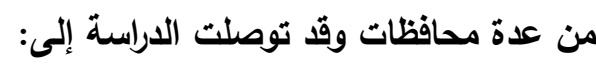
- - انخفاض مستوى الوعي بالمشكلات البيئية. - ت توجد فروق في إدراك التلاميذ في المراحل المختلفة لقضايا البيئة.

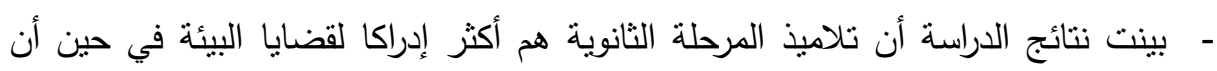
تلاميذ المرحلة الابتدائية هم أقل إدراكا لقضايا البيئة.

\section{ك}

- ل لما كانت الاتجاهات تعتبر من أهم مكونات الخبرة التي تحدد مدى قدرة الفرد على المساهمة في حل المشكلات البيئية والتصدي لها، كما تعتبر من منابع الطاقة الحقيقية

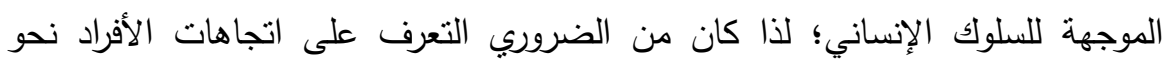
بيئتهم ومشكلاتها. 
- - ويري بعض المربين أن الحل الجزري لأزمة البيئة الراهنة يتطلب تغيرا كبيرا في اتجاهات الإنسان إزاء بيئته، بل إن بعضهم يرى أن الثورة البيئية التي نحن في حاجة إنها إليها إنما هي لئه

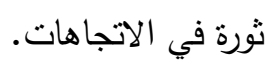

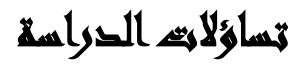

ا-ما اتجاهات الثباب السعودي بمدينة بريدة نحو المشكلات البيئية التي يتعرض لها

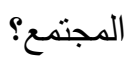

r-ما اتجاهات الثباب السعودي نحو مشكلات تلوث البيئة؟ r-ما اتجاهات الثباب السعودي نحو مشكلات استتزاف موارد البيئة؟

\section{أهمي التواسمة}

1أهمية مشكلات البيئة وتصاعد خطورتها واهميتها في مدينة بريدة بالسعودية r-أهية مرحلة الثباب لأنهم صناع الحاضر والمستقبل وأهيل

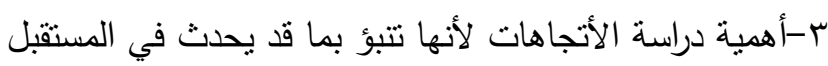

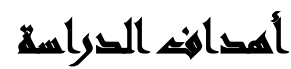

تسعى هذه الدراسة إلى التعرف على اتجاهات الثباب السعودي بمدينة بريدة نحو

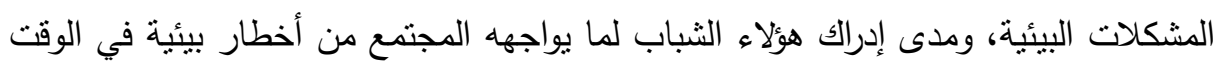
الحالي، ورؤية هؤلاء الثباب لكيفية التغلب على هذه المشاكل، وذلك من خلاه تحديد ما يلي: هدف رئيسي :دراسة اتجاهات الشباب نحو المشكلات البيئية. ويتفرع من هذا الهـف الرئيسي الأهداف الفرعية التالية: 1-دراسة اتجاهات الثباب نحو مشكلات النلوث البيئي r- دراسة اتجاهات الثباب نحو مشكلات استتزاف الموارد البيئية r-الخروج بمقترحات لتدعيم اتجاهات الثباب نحو مشكلات البيئة 


\section{نه}

تعد هذه الدراسة من الدراسات الوصفية حيث الدراسات المسحية الوصفية من أكثر طرق البحث شيوعا في دراسة الظواهر الانسانية والاجنماعية التي تستهدف قياس الاتجاهات والمعتقدات والميول لافراد المجتمع كذلك أنماط السلوك المختلفة في وصف وتفسير العلاقات المتبادلة بين هذه لعناصر بعضها ببعض وبيانات منهج الدراسة: تستعين الدراسة بمنهج المسح الاجتماعي عن طريق العينة أعتمدت الدراسة علي منهج المسح الاجتماعي وهو احد أساليب المنهج العلمي لمعرفة حقيقة الظاهرة الني تقوم الدراسة بمحاولة تقديم حلول لنلك الظاهرة . أن ذلك المنهج أعتبره الباحث اسلوب للتفكير لتتظيم الأفكار وعرضها وتحليلها للوصول

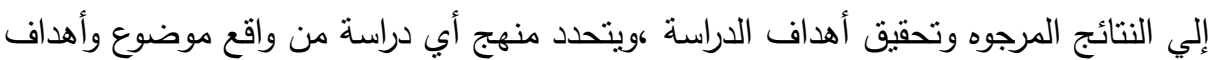
البحث والدراسة (محمد الغريب :البحث العلمي-التصميم والإجراءات ،دار نهضة الثرق

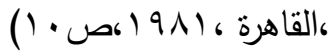
عينة الدراسة ومجتمع الدراسة: طبقت الدراسةعلي عينة من المدارس الثانوية للبنين والبنات من مدينة بريدة بلغ عدد العينة( جr ) مفردة من البنين والبنات هما: - مدرسة ثانوية فيصل بن فهر للبنين

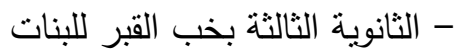

\section{مجالايت الصراسة}

أولاً: المجال البشري: ويقصد به الأفراد أو المبحوثين الذين أجريت عليهم الدراسة الميدانية- تم اختيار عينة عشوائية من طلبة المدارس الثانوية للبنين والبنات من مدينة بريدة،وقد شملت العينة (T Y T)مفردة ثانياً: المجال الجغرافي: ويقصد به الحدود الجغرافية لمجتمع البحث والأماكن التي تردد عليها الباحث لذلك جاءت عينة الدراسة المكانية من بعض مدارس مدينة بريدة 
- مدرسة ثانوية فيصل بن فهد للبنين

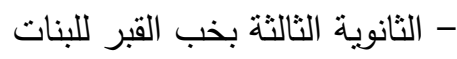
ثالثاً: المجال الزمني: ويقصد به المدة التي استغرقها الجانب الميداني للاراسة

\section{أسواهي التصراسة}

أدوات جمع البيانات: الأداة هي الوسيلة العلمية التي سوف يستخدها الباحث في جمع البيانات من مفردات المجتمع الذي يحدده. ومن المسلم به نجاح البحث في تحقيق أهدافه يتوقف على الاختيار الرشيد لأنسب

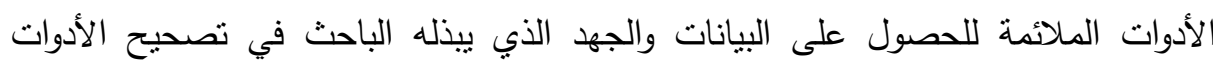
وتتظميها وجعلها على مستوى من الكفاءة. الاستبيان: استمارة استبيان للمشكلات البيئية للتعرف وملاحظة سلوكيات الثباب نحوها كما يستخدم دليل المقابلة ليتعرف على رؤية الثباب للمشكلات البيئية وذلك لتحديد اتجاهات الشباب نحو المشكلات البيئية.

والاستبيان هو أحد أدوات جمع البيانات وأكثرها استخدما وشيوعا في البحوث الاجتماعية

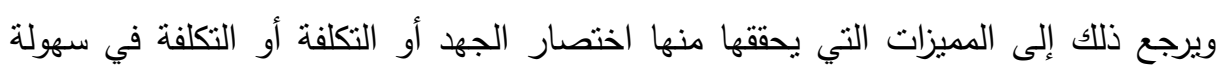

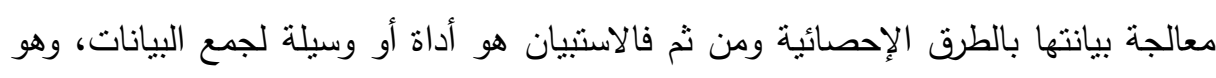

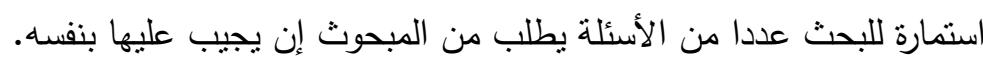

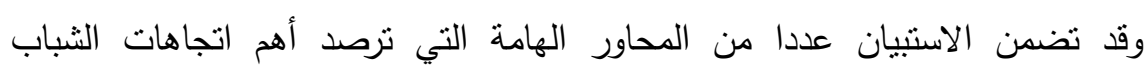

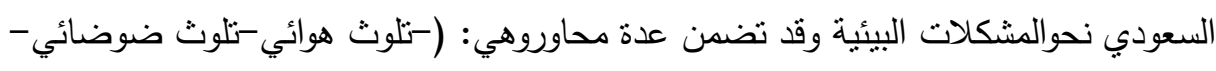

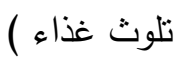
-استنزاف للمواردمثل (الماء-الكهرباء-التربة) - نوازن البيئي 
أولاً: صدق الاستبيان: يعتبر صدق الاستبيان شرطا أساسيا من شروط أدوات المقياس في قياس الظاهرة

1 - صدق المحكمين: تم عرض المقياس في صورته المبدئية علي (• ( من أعضاء هيئة

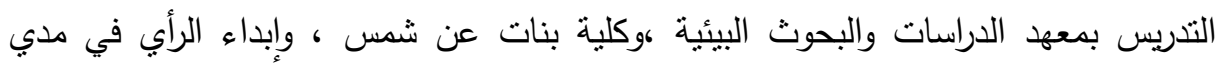

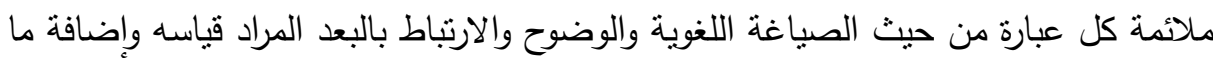
يبدونه سيادتهم من عبارات تخدم أب بعد من إبعاد الاستبيان ،وكذلك حذف الغير الملائم منها ،واعتمدت الباحثة علي نسبة اتفاق لا تقل عن (10، •) حيث تعتبر نسبة اتفاق المحكمين

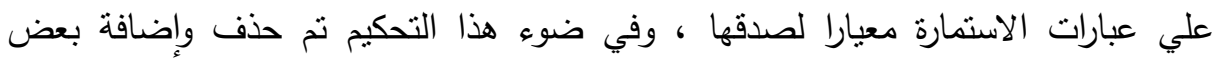
العبارات وتعديل بعض الصياغات حتى يكون الاستبيان محل ثقة في نتائجه وصدقه . ثانياً: الثبات يعد ثبات شرطا من شروط استخدام المقياس كوسيلة وفعالة ، ذلك لتحديد اتجاهات الثباب السعودي نحو المشكلات البيئية ينم حساب معاملات ثبات الدراسة بطريقة

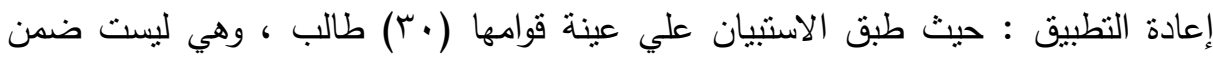

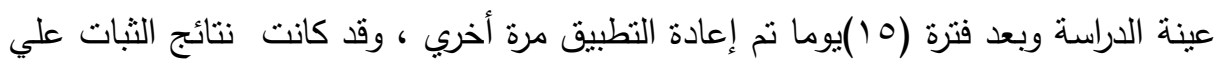

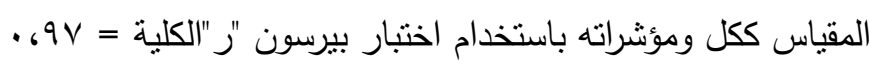
r ب-الاستيّيان- استمارة استبيان للمشكلات البيئية المعالجة الإحصائية: بعد نطبيق أدوات الدراسة وجدولة النتائج، نم استخدام أساليب إحصائية تنتاسب مع فروض الدراسة الحالية، وهذه الأساليب كالتالي: تم معالجة البيانات الأساسية من خلال الحاسب الآلي باستخدام برنامج ( spss) الحزم الإحصائية التالية:

$$
\begin{aligned}
& \text { 1- المتوسطات الحسابية. } \\
& \text { r- الانحرافات المعيارية. } \\
& \text { T.test r-اختبار } \\
& \text { ع -معامل ارتباط بيرسون. } \\
& \text { ه-المعالجة الاحصائية }
\end{aligned}
$$

$$
\text { المجلد السادس والثثاثون، الجزء الثاني، ديسمبر } 17 \text {. }
$$


عرض تحليلي للبيانات الإحصائية: أشنارت الدراسة الميدانية وتحليل البيانات إحصائيا إلي النتائج العامة للبحث: أثنار تحليل البيانات الاحصائية إلي عدد من النتائج العامة منها:

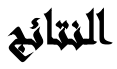

ا ـ شاهدت أحد زملائك يقطع زهور حديقة المدرسة

\begin{tabular}{|c|c|c|}
\hline نسبة & تكرار & \\
\hline$r \Lambda, r$ & $7 \varepsilon$ & تتزكه وشأنه \\
\hline 01,1 & $11 \mathrm{~V}$ & تحاول أن تقنعه بأضرار هذا العمل. \\
\hline 19,9 & «o & تقوم بتقليده \\
\hline $1 \cdots, \cdot$ & YYT & الاجمالى \\
\hline
\end{tabular}

يوضح الجدول السابق: ان اجابة سؤال شاهدت أحد زملائك يقطع زهور حديقة المدرسة كانت

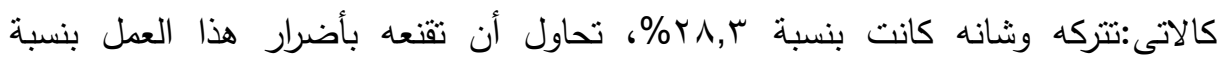

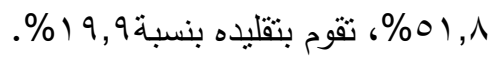

r ب بعد انتهاء اليوم الدراسي شاهلات مصابيح الكهرباء ،مضاءة،ماذا تفعل؟

\begin{tabular}{|c|c|c|}
\hline نسبة & تكرار & \\
\hline $0 \cdot, \cdot$ & $11 \pi$ & آقوم بغلق المصابيح \\
\hline $.2 Y 0, r$ & ov & آطلب من أحد الزملاء إطفاء النور \\
\hline$r \varepsilon, \Lambda$ & 07 & ابلخ الإدارة عنهم \\
\hline $1 \cdots, \cdot$ & YYY & 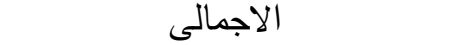 \\
\hline
\end{tabular}

يوضح الجدول السابق: ان أقوم بغلق المصابيح بعد انتهاء اليوم الدراسي كانت بنسبة

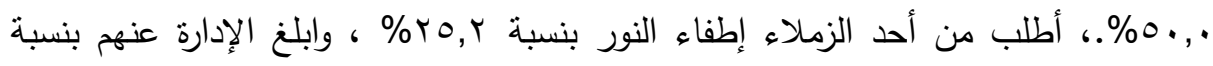
.\% $r \leqslant, \wedge$

r. إذا شاهدت زملائك يكتبون علي جدران الفصل؟

\begin{tabular}{|c|c|c|}
\hline نسببة & تكرار & \\
\hline$\sum 7,0$ & 1.0 & أوضح لهم آن هذا نتويه لجدران المدرسة. \\
\hline$\varepsilon 1,7$ & $9 \leq$ & أبلغ عنهم إدارة المدرسة لاتخاذ اللازم \\
\hline 11,9 & TV & لا اهتم \\
\hline $1 \cdots, \cdot$ & TYT & الاجمالى الى \\
\hline
\end{tabular}


يوضح الجدول السابق: أوضح لهم أن هذا تشويه لجدران المدرسة إذا شاهدت زملائك يكتبون

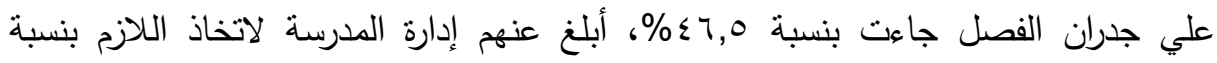

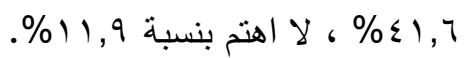

ع -إذا رأيت بعض الأشخاص يحرقون القمامة خارج سور المدرسة فماذا تفعل؟

\begin{tabular}{|c|c|c|}
\hline نسبة & تكرار & \\
\hline$\Gamma \leqslant, 0$ & $\mathrm{~V} \wedge$ & انصحهم بعدم حرق القمامة لأنها تلوث الهواء \\
\hline$\leqslant 0,7$ & $1 . r$ & ابلخ إدارة المدرسة. \\
\hline 19,9 & $\leqslant 0$ & اتزكهم واذهب \\
\hline $1 \cdots, \cdot$ & TYY & الاجمالى \\
\hline
\end{tabular}

يوضح الجدول السابق: جاءت ابلغ إدارة المدرسة في المرتبة الاولى حيث كانت النسبة

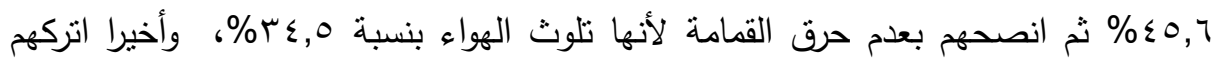

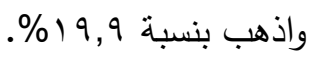

ه-لو شاهلت حنفية المياه في المدرسة مفتوحة دون استخدام؟

\begin{tabular}{|c|c|c|}
\hline نسبة & تكرار & \\
\hline$r V, q$ & $7 \pi$ & ابلغ العامل المسئول ليغلقها \\
\hline$\varepsilon 0,1$ & $1 \cdot Y$ & آقوح بغلقها \\
\hline$T V, \cdot$ & 71 & لا آهتح. \\
\hline $1 \cdots, \cdot$ & TYT & الاجمالي \\
\hline
\end{tabular}

يوضح الجدول السابق: جاءت في المرتبة الاولى أقوم بغلقها بنسبة إه؛\% ، ابلغ العامل

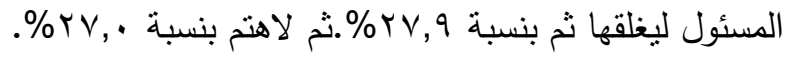

צ-لو شاهلات أي إنسان بلقي بالقمامة الخاصة به بجوار سور المدرسة؟

\begin{tabular}{|c|c|c|}
\hline نسبة & تكرار & \\
\hline$\{0,1$ & $1 \cdot r$ & أجمعها \\
\hline$\Gamma \varepsilon, 0$ & $\overline{V A}$ & أطلب منه جمعها \\
\hline$r_{\cdot, \Sigma}$ & $\sum 7$ & لا أهتم \\
\hline $1 \cdots, \cdot$ & TYT & الاجمالى \\
\hline
\end{tabular}

يوضح الجدول السابق: جاءت أجمعها لو شاهدت أب إنسان يلقي بالقمامة الخاصة به بجوار

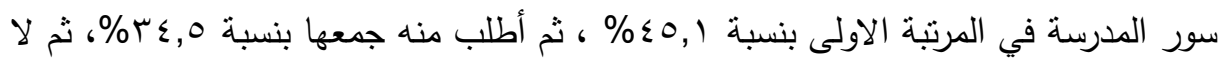

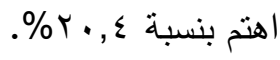


V-ما رأيك في تحويل المخلفات الثانوية في الصناعة إلي منتجات تدخل في صناعات

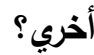

\begin{tabular}{|c|c|c|}
\hline نسبة & تكرار & \\
\hline YI,Y & $\sum \Lambda$ & لا يـ2 \\
\hline $01, \pi$ & 117 & مهم الى حذ ما \\
\hline$r V, \varepsilon$ & $T Y$ & مهر جدا \\
\hline $1 \cdots$, & TYT & الاجمالى \\
\hline
\end{tabular}

يوضح الجدول السابق: ما رأيك في تحويل المخلفات الثانوية في الصناعة إلي منتجات تدخل

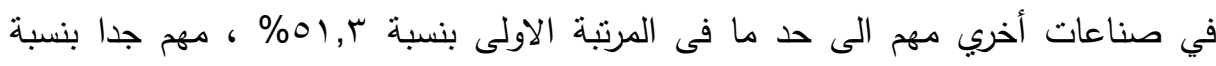

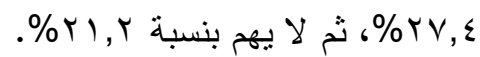

^- ما رأيك في المحافظة علي الغطاء الأخضر وتتميته وخصوصا في الاراضي الصحراوية

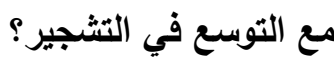

\begin{tabular}{|c|c|c|}
\hline نسبةً & تكرار & \\
\hline $.5 Y r, 0$ & or & لا يهر \\
\hline$\xi Y, \cdot$ & 90 & مهم الى حد ما \\
\hline$\Gamma \varepsilon, 0$ & $\vee \wedge$ & مهر جدا \\
\hline $1 \cdots, \cdot$ & TYT & الاجمالي \\
\hline
\end{tabular}

يوضح الجدول السابق: ما رأيك في المحافظة علي الغطاء الأخضر وتتميته وخصوصا في الاراضي الصحراوية مع التوسع في التتجير مهم الى حد ما فى المرنبة الاولى بنسبة

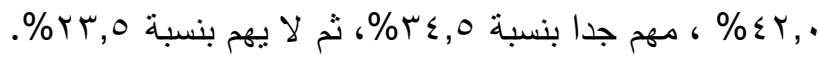


حاتم عبد المنعم أحمد وآخرون

يوضح الجدول: انه يوجد فروق بين الذكور والاناث ذات دلالة إحصائية في مقياس المشكلات

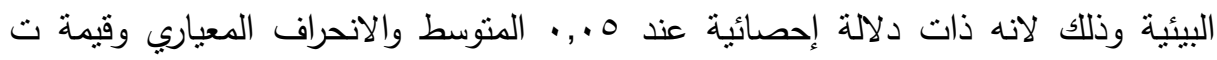
والدلالة للمقارنة بين الذكور والإناث في محاور المقياس

\begin{tabular}{|c|c|c|c|c|c|c|}
\hline الدلالة & قيمة ت & الانحيراف & المتوسط & العدد & النوع & \\
\hline \multirow{2}{*}{$\cdot, \cdots$} & \multirow{2}{*}{$r, 011$} & $r, 1 \ldots .01$ & $10,79 \cdot r$ & $11 \pi$ & ذكور & \multirow{2}{*}{ تلوث بيئي } \\
\hline & & $1,9 \wedge 1 \vee$ & $17,7 \leq 7$ & $11 \pi$ & إناث & \\
\hline \multirow{2}{*}{$\cdot, \cdots}$, & \multirow{2}{*}{,$Y \backslash \uparrow$} & $1,117.0$ & $0, \varepsilon 01 \mu$ & $11 \pi$ & إناث & \multirow{2}{*}{ تلوث هوائي } \\
\hline & & $\cdot, 9 Y \backslash \leq 7$ & $\varepsilon, \wedge \mid \leqslant Y$ & $11 \pi$ & ذكور & \\
\hline \multirow{2}{*}{$\cdot, \cdots 1$} & \multirow{2}{*}{$0, \vee \wedge \mu$} & 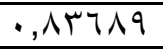 & $r, 10 . \varepsilon$ & $11 \pi$ & ذكور & \multirow{2}{*}{ تلوث ضوضائي } \\
\hline & &., $7 / 91 \mathrm{~V}$ & $r, V \backslash 7 \wedge$ & $11 \pi$ & إناث & \\
\hline \multirow{2}{*}{$\cdot, \varepsilon$} & \multirow{2}{*}{$\cdot, \wedge r q$} & $r, 1 M 0 \leqslant r$ & $1 \mu, \wedge 9 \mu \wedge$ & $11 \pi$ & ذكور & \multirow{2}{*}{ تلوث غذائي } \\
\hline & & $r, 1 \leq \varepsilon r y$ & $I \varepsilon, I M Y V$ & $11 \pi$ & إناث & \\
\hline \multirow{2}{*}{$\cdot, \cdots}$, & \multirow{2}{*}{$7,7 \vee \leq$} & Y,I To & $1 \Gamma, \wedge 9 \varepsilon$ & $11 \pi$ & ذكور & \multirow{2}{*}{ استنزاف موارد } \\
\hline & & $r, 1 \leq \varepsilon$ & $1 \varepsilon, 1 \Gamma$ & 111 & إناث & \\
\hline \multirow{2}{*}{$\cdot, \cdot r$} & \multirow{2}{*}{$r, r \vee q$} & $r, \wedge r q$ & r1,991 & $11 \pi$ & ذكور & \multirow{2}{*}{ توازن بيئي } \\
\hline & & Y,TIV & $r \leq, Y 9 T$ & 111 & إناث & \\
\hline \multirow{2}{*}{$\cdot, \cdots}$, & \multirow{2}{*}{ ת שזT 0, } & $r, r . q$ & $r r, \Sigma 79$ & 111 & ذكور & \multirow{2}{*}{ إجمالى المقياس } \\
\hline & & $r, \wedge \circ r$ & $\Gamma \varepsilon, 00 \wedge$ & 114 & إناث & \\
\hline
\end{tabular}
يشير الجدول السابق انه: - يوجد فرق دال إحصائياً عند مستوى دلالة (0., ·) في منوسط درجات التناميذ لابعاد المقياس ولاختبار صحة هذه التساؤلات تم حساب المتوسط الحسابي والانحراف المعياري وقيمة (ت) للطلاب مجموعة البحث .ووجد انه توجد فروق دالة احصائيا بين الذكور والاناث في كل ابعاد المقياس الا التلوث الغذائى فلا توجد فيه اي فروق بين الجنسين .

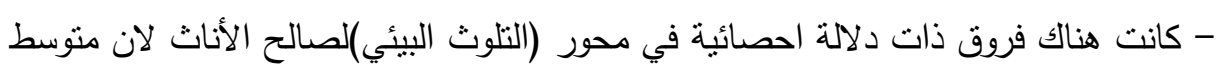

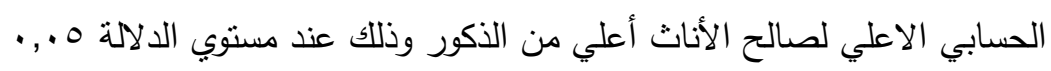

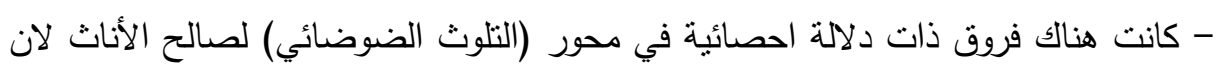

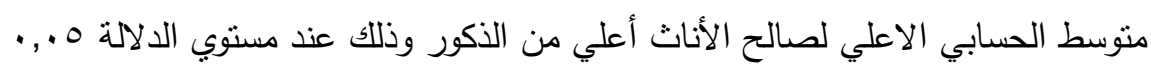

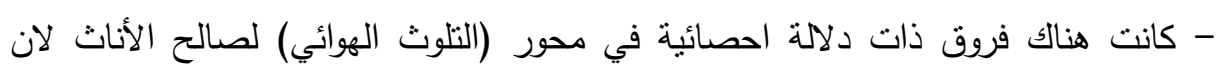

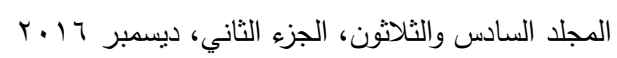


منتوسط الحسابي الاعلي لصالح الأناث أعلي من الذكور وذلك عند مستوي الدلالة ه.,.

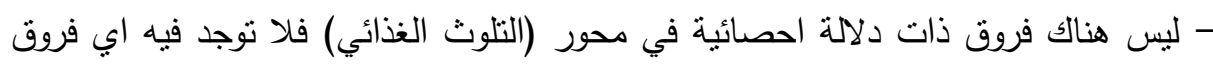
بين الجنسين - كانت هناك فروق ذات دلالة احصائية في محور (استنزاف الموارد) لصالح الأناث لان

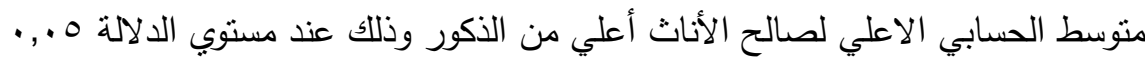

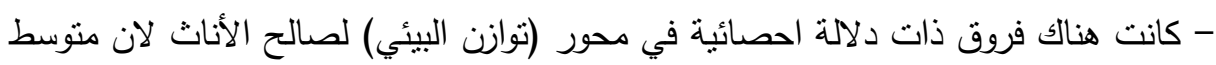
الحسابي الاعلي لصالح الأناث أعلي من الذكور وذلك عند مستوي الدلالة هـ هـ ـ.

- اتضح من النتائج أن هناك أدراك لاى الثباب السعودي بمدينة بريدة بالخطار المحيطة به الهيه الناتجة عن المشكلات البيئية ومنها - أوضحت النتائج ان هنالك معرفة لدي الثباب السعودي بمدينة بريدة بوجود مشكلات بيئية تحيط به وتمثل خطرا عليه - أوضحت النتائج ان هناك اتجاهات من كلا الجنسين من الثباب تجاه الشككلات التي

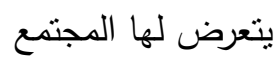
- أوضحت النتائج انه يوجد فرق دال إحصائيا عند مستوي دلالة (0. . • ) في منوسط درجات التلاميذ لبعد الذكور والاناث ولاختبارصحة هذا الفرض نم حساب المتوسط الحسابي

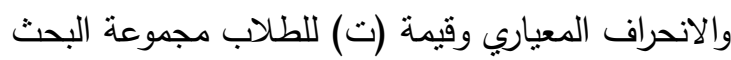

\section{ing}

في ضوء ما تم عرضه في هذه الدراسة من مفاهيم نظرية ونتائج ميدانية توصي هذه

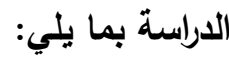
- أن تشتنل المناهج الدراسية علي مشكلات بيئية واقعية بمكن عرضها بطريقة مناسبة ومترابطة بحيث يمكن تقديمها وفقا لأهميتها وخطورتها علي المجتمع. - زيادة فرص النشاط البيئي في مناهج المواد الدراسية المختلفة لممارستها داخل المدرسة وهية وخارجها. 
- ضرورة ربط المدرسة بالمجتمع المحلي المحيط بها وبمشكلاته البيئية وعليها إن تشارك وتنساهم في حل هذه المشكلات.

- ضرورة الأخذ في الاعتبار أساليب تتمية السلوكيات البيئية في عمليات التعليم والتعلم داخل المدرسة من اجل الحفاظ علي البيئة ومواردها لدي التلاميذ.

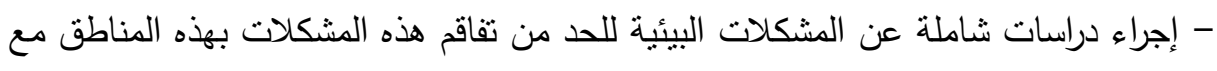

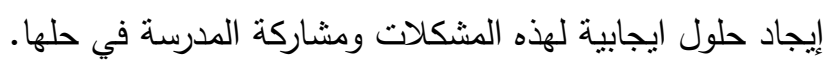

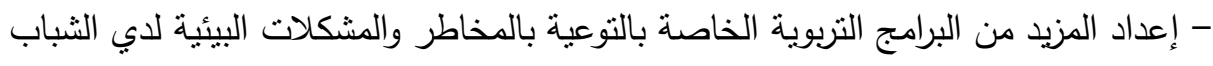
- الحفاظ على العمليات البيئية والأنظمة الحيوية الأساسية التي نتوقف عليها بليها عملية التتمية ومن أمثلتها نظافة الهواء،نظافة الماء، المحافظة على العشائر النباتية والحيوانية ذات التئه الأهمية الخاصة( الأعثاب البحرية- الثعب المرجانية- المستتقعات-الغابات) - المحافظة على الغطاء الأخضر وتتميته وخصوصاً في الأراضي الصحراوية مع التوسع في الأبه •التشجير

- المحافظة على النتوع الحيوي من خلا بنوك الموروثات والمحميات الطبيعية وحدائق النباتات. - إيقاف التعديات على الأراضي الزراعية وتتمية ثروة البحيرات من طيور وأسماك. - المحافظة على المراعي وتتمينها والحد من الرعي الجائر والتحطيب.

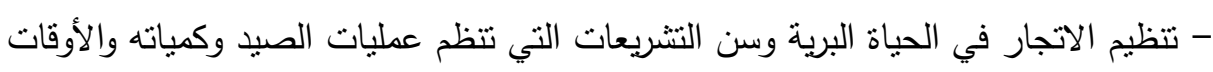
المسموح بالصبد فيها والأدوات التي تستخدم في الصيد.

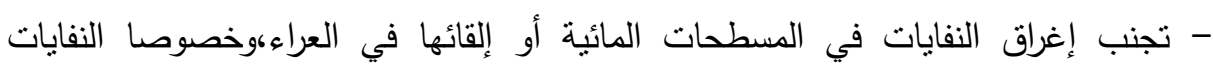
النووية والتنفيذ الجاد لمعاهدة خطر التجارب النووية حماية للبيئة البحرية من التلوث.

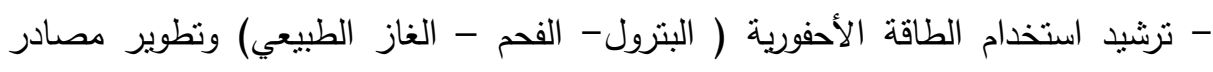
الطاقة البديلة مثل طاقة الرياح والطاقة الثمسية وطاقة الكتلة الحيوية. 


\section{المراجية}

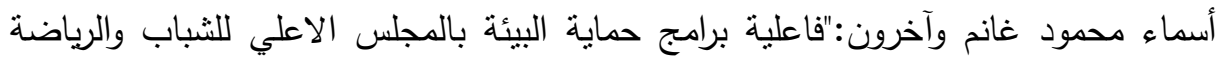
لتتمية الاتجاهات البيئية لدي الطلائع، مجلة العلوم البيئية، المجلد السادس،

$$
\text { العدد الثاني، يونيو ل ... }
$$

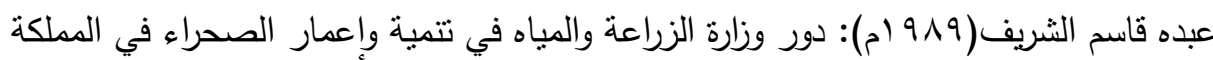

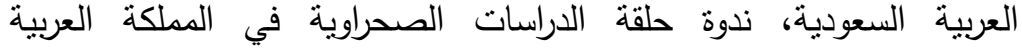
السعودية: مجالاتها والمهتمون بها، جامعة الملك سعود

ابراهيم بن مبارك الجوبر (0 1 (هـ): الثبا وقضاياه المعاصرة، مكتبة العبيكان، السعودية سليمان محمد العقيلي،وبشيرمحمود حرار( •99 (): تلوث الهواء ،مكتب التربية العربي لدول

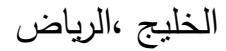

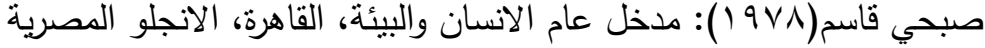

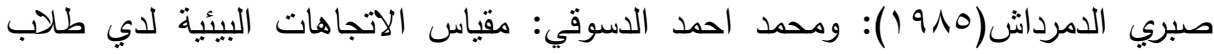

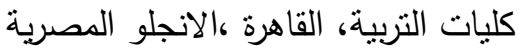

فتحي عبد المقصود الديب، وآخرون( •99 (1): أبعاد التربية البيئية في المناهج الدربة الدراسية لمعلم المدرسة الابندائية في الدول العربية المركز القومي للبحوث التربوية والتنمية التية التية

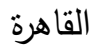

فادية مغيث( •99 (19): مشكلة تلوث البيئة ودور التربية في مواجهتها، كلية التربية

Ahmed Melha, ( juin 2001) : Les Enjeux Environnementaux en Algérie, Population initiatives for peace, $\mathrm{p} 150$

Bull , James Nickell (1992): The Effect of Participation in an Environmental Action Problem -Solving Skills of inner city students, (P.H.D) University of Michigan,

Burdon, D., (1971), Exploitation of groundwater for agricultural production in arid lands, in W. McGinnies B. Goldman, and P. Paylore, (eds.), Food, Fiber and the Arid Lands, The University of Arizona Press, Tucson, Arizona

Berger ,I,E (1997) :The Demographics of Recycling Behavior Environmental Behavior 
حاتم عبد المنعم أحمد وآخرون

Cohen ,Jeffrrey J:Fish Marian C(1993):, Handbook of school Based intervention resoiving student promating healthy education environments social and behavioral science serics

\title{
SAUDI YOUTH ATTITUDES TOWARDS ENVIRONMENTAL PROBLEMS ON THE STUDY A SAMPLE OF SECONDARY SCHOOL STUDENTS IN THE CITY OF BURAIDAH
}

\author{
Ahmed, H. A. ${ }^{(1)}$; Awad, M. I. ${ }^{(1)}$; Al-Tuwaijri, A. M. ${ }^{(2)}$ \\ and Reshoudi. A. S. M. \\ 1) Institute of Environmental Studies and Research, Ain Shams \\ University.2) Qassim University
}

\begin{abstract}
Taadalatjahat according to the opinion of scientists of the most important components of the experience that determine an individual's ability to contribute to the solution of environmental problems and address them, as we hold the real sources of energy directed human behavior so it was necessary to identify the trends of individuals about their environment and its problems.

So some scholars of Education believes that the best solution to the current crisis environment requires a significant change in human attitudes about the environment, but that some of them believes that the environmental revolution, which we desperately need it but a revolution in attitudes, so conservative Ali become the ecological balance of the best way to avoid the risk of these problems If we are to maintain the ecological balance must stop the unfair interference of man in environmental activities.
\end{abstract}

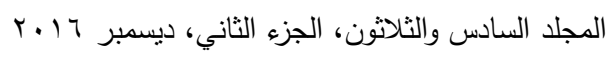


That environmental problems can not be dealt with through technological procedures These important though measures alone are not sufficient to meet these environmental problems The problems should be resolved in the framework of social, economic and cultural causes and through a comprehensive action plan are discipline in the development patterns in the lifestyles of individuals and in their behavior and there are many environmental problems

Interested in environmental issues and problems have realized as education scientists realized that the most important ways feasible in the face of environmental problems lies in human numbers who understands the environment and conscious surroundings from the dangers capable of positive contribution to the preservation and development for the benefit of the environment and provide a decent life for man himself, this is what consideration by this study

. Objectives of the study is to identify the Saudi youth trends in Buraidah about environmental problems and perceptions of what these young people facing the community of environmental hazards at the moment, and to see these young people on how to overcome these problems

The study is based on the social survey method through the sample, consisting of 226 students from area schools Buraidah Saudi Arabia And research tool used is a questionnaire to environmental problems

And they've made them the basic analysis and study concluded a number of important results such as:

- atdh From the results that there is realization among Saudi youth in Buraidah dangers surrounding the resulting environmental problems, including the Results -aodan I know that there are young Saudi city of Buraidah existence of environmental problems is surrounded by and pose a threat to him 


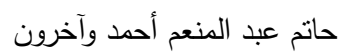

Results -aodan that there are trends of both sexes of young people towards the problems faced by society: As well as a number of recommendations including:

The curriculum includes realistic environmental problems can be displayed appropriate and coherent way so that it can be submitted according to the importance and danger to the community

Increase the chances of environmental activism in different subjects, approaches to practice inside and outside the school 